\title{
Manejo del insomnio en el adulto
}

\section{Management of insomnia in the adult}

Dra. Andrina Valverde Jiménez ${ }^{1}$ Dra. Ana Catalina Agüero Sánchez²Dra. Juliana Salazar Mayorga ${ }^{3}$

1,2 y 3 Médico general. Trabajador independiente, San José Costa Rica.

Contacto: andrinavalverde@gmail.com

\section{Resumen}

El insomnio es un trastorno del sueño, se clasifica según el tiempo de evolución, según si se asocia a otra patología y según su severidad. El diagnóstico es principalmente clínico y no amerita estrictamente estudios complementarios como exámenes de laboratorio y gabinete. El tratamiento no farmacológico incluye la terapia de comportamiento cognitivo, las medidas de higiene del sueño, control de estímulos y técnicas de relajación; mientras que el tratamiento farmacológico debe ser individualizado para cada paciente y podría involucrar el uso benzodiacepinas, compuestos Z, antagonistas de melatonina o antidepresivos tricíclicos. Existe un gran impacto en la calidad de vida y salud física-mental en los pacientes que sufren de insomnio.

Palabras clave:

Trastornos del Inicio y del Mantenimiento del Sueño, Trastornos del sueño-vigilia, Higiene del sueño, Salud mental

\section{Abstract}

Insomnia is a disorder of sleep, it is classified according to the time of evolution, according to whether it is associated with another pathology and according to its severity. The diagnosis is mainly clinical and complementary studies are not strictly warrant, such as laboratory and cabinet examinations. Non-pharmacological treatment includes cognitive behavior therapy, sleep hygiene measures, stimulus control, and relaxation techniques; while pharmacological treatment must be individualized for each patient, and could involve the use of benzodiazepines, $Z$ compounds, melatonin antagonists or tricyclic antidepressants. There is a great impact on the quality of life and mental physical health in

Recibido: $03 / \mathrm{mrz} / 2020$ patients suffering from insomnia.

Aceptado: $16 /$ nov/2020

Publicado: $15 /$ dic/2020
Keywords:

Sleep Initiation and Maintenance Disorders, Sleep Wake Disorders, Sleep hygiene, Mental health. 


\section{INTRODUCCIÓN}

Los trastornos del sueño son motivo de consulta común para los médicos y profesionales en salud, por lo que es importante conocer esta patología, y así, lograr un abordaje oportuno para los pacientes. Se ha estudiado que la privación de sueño conlleva consecuencias negativas en la estabilidad emocional de las personas, aún sin tener asociada alguna patología psiquiátrica $(1,2)$. El sueño cumple en el organismo humano una función reparadora, de gran importancia para la estabilidad afectiva, laboral, y en general, el funcionamiento en la vida diaria y distintos aspectos de salud en las personas $(3,4)$.

El insomnio se define como la dificultad de conciliar el sueño o mantenerse dormido, despertares nocturnos o despertar muy temprano; esto asociado a la insatisfacción o preocupación por la falta de sueño, también asociado a cambios de humor, irritabilidad, fatiga, somnolencia, mal desempeño laboral y/o en la vida social, implicaciones negativas en memoria, concentración y/o atención (5-8).

El objetivo de la presente revisión es integrar información científica actualizada sobre el diagnóstico, clasificación, manejo no farmacológico y farmacológico del insomnio que les permita a los profesionales de la salud comprender puntualmente esta patología y brindarles a estos pacientes un manejo integral, acertado y oportuno que contribuya a mejorar su calidad de vida. Lo anterior debido a que esta es una patología que genera gran porcentaje de las consultas clínicas de un médico general, en situaciones actuales de estrés se vuelve fundamental conocer el tema.

\section{MÉTODO}

Para esta revisión bibliográfica se realizan búsquedas de textos científicos publicados en bases de datos como EBSco, UpToDate, Medline y Google Scholar. La búsqueda se limitó por medio del uso de las palabras clave: "trastornos del sueño", "insomnio", "manejo de insomnio", "tratamiento del insomnio", "ciclo de sueño". Se tomaron en cuenta textos escritos en idioma español e inglés con información actualizada y relevante en artículos científicos publicados entre los años 2015 y 2020. Posteriormente se filtraron los artículos obtenidos según su importancia científica. Se revisaron los resúmenes, conclusiones y en algunos casos los artículos completos, según llamó el interés de los investigadores; obteniendo así los documentos que incluían la información necesaria para el cumplimiento de los objetivos y ampliación de información.

\section{EPIDEMIOLOGÍA}

En la población general, la prevalencia del insomnio se encuentra entre el 20-36\% y en pacientes diagnosticados con algún trastorno psiquiátrico aumenta a un $40-90 \%(1,9)$.

Para diciembre del 2019, en Estados Unidos se reportaron alrededor de cinco millones de consultas al año por insomnio y además, alrededor del $70 \%$ de la población reportó haber sufrido insomnio en algún momento de la vida, siendo más frecuente en mujeres y mayores de 65 años. Es una patología común de los países industrializados y además es un importante motivo de consulta en el primer nivel de atención (7-9).

\section{COMORBILIDADES Y FACTORES DE RIESGO}

Más del $50 \%$ de los pacientes que consultan por insomnio tienen uno o más factores de riesgo o comorbilidades asociadas, por lo que se deben tomar en cuenta en el historial clínico de los pacientes. Entre las características de estos pacientes se encuentran los pacientes desempleados, divorciados, viudos, separados de su pareja sentimental y con bajo nivel socioeconómico $(7,9)$.

Entre los factores de riesgo intrínsecos para el insomnio que se mencionan en la literatura se encuentran: ser mayor de 65 años, género femenino (específicamente en perimenopausia o postmenopausia), haber sufrido un episodio de insomnio previo, antecedentes heredofamiliares positivos de insomnio y predisposición genética $(5,7)$

Entre las comorbilidades más comunes se encuentran $(6,7,9)$.

1. Enfermedades psiquiátricas (depresión, ansiedad, uso y abuso de sustancias, síndrome de estrés postrau mático, estrés psicológico).

2. Condiciones médicas (enfermedades pulmonares, hipertensión arterial, diabetes, pacientes oncológicos, dolor crónico, falla cardíaca, enfermedad de Parkinson, demencia tipo Alzheimer, estrés físico)

3. Medicamentos

a. Estimulantes del sistema nervioso central: cafeína, metilfenida to, anfetaminas. 


\section{CIENCIA@SALUD}

b. Depresores del sistema nervioso central: uso-abuso de bebidas alcohólicas.

c. Estimulantes del sistema respiratorio como teofilina, supresores del apetito, antidepresivos, beta bloqueadores, glucocorticoides, alcohol, tabaco, descongestionantes nasales.

4. Otros desórdenes del sueño: apnea del sueño, síndrome de piernas inquietas, etc.

\section{SÍNTOMAS}

La mayoría de los pacientes aqueja sintomatología diurna, por ejemplo: hipersomnolencia, alteración en la atención, concentración o memoria, fatiga, falta de motivación e iniciativa, también irritabilidad o alteraciones del humor. Algunos síntomas físicos tales como cefalea, fotofobia, alteraciones del tracto gastrointestinal y tensión pueden presentarse con frecuencia. Es frecuente que el género femenino aqueje entre 2 o 3 síntomas, mientras que los hombres normalmente aquejan durante la consulta un solo síntoma $(1,5,8)$.

\section{DIAGNÓSTICO}

El diagnóstico de esta patología es clínico, amerita una buena historia clínica para descartar los diferentes diagnósticos diferenciales y además para determinar con exactitud la causa y factores contribuyentes al insomnio $(6,7)$.

Para establecer el diagnóstico se requiere de tres criterios $(7,8)$ :

a. Dificultad persistente del sueño

b. Oportunidad adecuada para dormir

c. Disfunción diurna asociada

El médico puede solicitarle al paciente un diario de sueño de al menos 7 días y 7 noches, muy útil en pacientes que tienden a exagerar sus síntomas. En el diario se incluyen las medidas de higiene que practica el paciente, los factores ambientales que influyen sobre el sueño del paciente, horario de siestas durante el día, consumo de cafeína y/o ingesta alcohólica, uso de medicamentos y descripción de las actividades que realiza el paciente en el dormitorio antes de acostarse a dormir (6, 7).

\section{DIAGNÓSTICO DIFERENCIAL}

Existen otros trastornos del sueño, que algunas veces pueden ser diagnosticados erróneamente como insomnio, por lo que es importante tenerlos en consideración. Entre estos se encuentra, apnea obstructiva del sueño, que en un $30 \%$ de los casos puede coexistir con insomnio por lo que, debe ser considerado en el diagnóstico diferencial de los pacientes con insomnio crónico resistente al tratamiento $(6,7,9)$.

También deben considerarse en el diferencial los trastornos de sueño relacionados con el movimiento como el síndrome de piernas inquietas y los movimientos periódicos de las extremidades del sueño, los cuales pueden producir fragmentación y dificultad para mantener el sueño $(6,7)$.

Los trastornos del ciclo circadiano como el jet-lag también deben considerarse dentro del diagnóstico diferencial, ya que pueden causar insomnio de curso agudo, al igual que los trabajos en turnos nocturnos, donde se dificulta la conciliación del sueño dado que durante el día, mientras estos pacientes duermen, el ciclo circadiano promueve la vigilia $(6,7)$.

\section{CLASIFICACIÓN}

Entre los trastornos del sueño se encuentran: trastornos del sueño relacionados con trastornos respiratorios, trastornos centrales de hipersomnolencia, parasomnias, trastornos del sueño relacionados con movimiento, trastornos del ciclo circadiano sueño-vigilia, e insomnio (6; 7). En este artículo se desarrollará únicamente el insomnio, por lo que la clasificación siguiente es solamente referente a este último.

Según causa subyacente: (10)

- Insomnio primario: aquel insomnio en el que no se ha identificado una causa subyacente o cuando ya se descartaron otras patologías.

- Insomnio secundario es el más frecuente y se asocia con trastornos psiquiátricos, médicos, uso de medi camentos y otros trastornos primarios del sueño.

Según el tiempo de evolución (8): 
- Insomnio transitorio: cuando dura menos de una semana.

- Insomnio agudo: se caracteriza por estar presente en un período entre 1 y 3 semanas, en el que se identi fica claramente una causa o agente estresor, si se elimina el agente causal se revierte el insomnio. Insomnio crónico: es el que se presenta al menos tres veces por semana durante al menos cuatro sema nas, algunos pacientes sí logran definir el momento de inicio del insomnio y asociarlo con algún agente causal, pero en otros pacientes es difícil determinarlo.

Según la severidad (9):

Insomnio leve: el paciente aqueja tiempo insuficiente de descanso nocturno, o no sentirse descansado después de una noche habitual de sueño.

Insomnio moderado: es cuando el paciente aqueja los síntomas de un insomnio leve y además hay disfun ción social u ocupacional.

Insomnio severo: el paciente tiene una disfunción completa, además de sentirse ansioso, irritable, fatiga do y muy cansado durante el día.

\section{MANEJO}

Los objetivos en el manejo del insomnio se basan en mejorar la calidad del sueño: en cuanto a tiempo (al menos 6 horas de sueño reparador), disminución de despertares nocturnos y latencia del sueño. Para cumplir con dicho objetivo se pueden emplear medidas farmacológicas y/o no farmacológicas. El médico tratante debe individualizar el manejo a cada paciente y adaptarlo a su condición y estilo de vida $(6,8)$.

\section{A) Tratamiento no farmacológico}

Dentro de las medidas no farmacológicas que se pueden practicar en pacientes con insomnio se encuentran: la terapia de comportamiento cognitivo, las medidas de higiene del sueño, control de estímulos, y las técnicas de relajación $(4,9,11,12)$

\section{Terapia cognitivo conductual $(9,13)$}

Es la primera línea de tratamiento para el insomnio crónico, existen estudios que afirman el éxito en el tratamiento cuando se aplica en conjunto la terapia de comportamiento cognitivo y el tratamiento farmacológico.

Este tratamiento se realiza de manera individual o grupal en sesiones que pueden ir de 4 a 8 . Esto incluye:

A. Establecer horario de hora de dormir y hora de despertar los sietes días de la semana.

B. Limitar el tiempo en la cama.

C. Incentivar el uso de la cama solo para dormir o mantener relaciones sexuales. Salir de la cama si aumenta la ansie dad al no concebir el sueño.

D. Medidas de higiene del sueño.

El tratamiento va dirigido a las siguientes metas:

A. Disminuir la ansiedad y/o frustración al insomnio.

B. Disminuir las expectativas con respecto a las horas de sueño.

C. Mitigar mitos con respecto a los efectos del insomnio.

D. Relajación muscular y meditación.

\section{Medidas de higiene del sueño (4,11):}

I. Ir a la cama solo en caso de que se tenga sueño.

II. Levantarse todos los días, incluso los fines de semana o los días libre, a la misma hora.

III. Pasar el mayor tiempo posible durante el día en lugares abiertos, al aire libre y ojalá en contacto con la naturaleza.

IV. Evitar quedarse en la cama sin estar dormido (a) más tiempo del necesario. 
V. Evitar las siestas durante el día.

VI. Reducir o eliminar el consumo de alcohol, cafeína o hipnóticos.

VII. Evitar comidas copiosas (altas en grasa o proteínas) antes de acostarse, cenar 3 horas antes de la hora planeada para dormir.

VIII. Mantener condiciones ambientales adecuadas para dormir, entre ellas: buena temperatura de la habitación, lugar ventilado, libre de ruidos y luz.

IX. Evitar actividades estresantes en las horas previas de acostarse.

X. Realizar un ejercicio físico moderado al final de la tarde.

XI. Practicar ejercicios de relajación antes de acostarse.

XII. Evitar el uso de pantallas, computador, televisor y celular una hora antes de acostarse.

XIII. Tomar baños de agua a temperatura corporal por su efecto relajante 3 horas previo a la hora planeada para dor mir.

\section{Control de estímulos}

Esta terapia se brinda debido a que existe una razón teórica de que el insomnio es una respuesta condicionada a las señales que percibe el paciente temporalmente al acostarse y lo que percibe en el ambiente de su dormitorio, por eso es que se logra asociar la cama y el dormitorio con el inicio del sueño al reducir las actividades incompatibles con el sueño que sirven de señal detonante del insomnio $(9,11)$

\section{Técnicas de relajación}

Se recomiendan las técnicas de relajación porque se ha visto que los pacientes con insomnio se mantienen con altos niveles de excitación fisiológica y cognitiva, durante las 24 horas del día, por lo que se intenta disminuir la excitación por medio de técnicas de relajación. $(4,9)$.

\section{B) Tratamiento farmacológico}

El manejo farmacológico es lo más apropiado para el insomnio agudo y debe estar acompañado de medidas no farmacológicas para el insomnio crónico $(9,12)$.

Algunos de los medicamentos que aprueba la FDA (Food and Drugs Administration) para el tratamiento del insomnio son:

-Benzodiazepinas: que disminuyen el tiempo para inicio del sueño, el número de despertares nocturnos y aumentan la cantidad de tiempo de sueño por medio de la modificación en la arquitectura de las fases del sueño. Son adecuados para tratamiento de insomnio agudo, deben ser utilizados en pacientes adultos jóvenes y por períodos cortos $(8,9,14)$

-No benzodiacepinas o compuestos Z: Zoplicona, Zolpidem, Zaleplon, Eszoplicone, son igual de efectivos que las benzodiacepinas, sin modificar la arquitectura de las fases del sueño como sí lo hacen las benzodiacepinas $(8,9)$.

-Agonistas de receptor de melatonina: Ramelteon mejora la calidad de sueño y disminuye el tiempo para conciliar el sueño al ser un agonista selectivo de los receptores de melatonina, sin afinidad por los receptores de benzodiacepinas, dopamina ni los de serotonina, además es un medicamento efectivo en adultos mayores $(8,9,13)$.

-Antidepresivos tricíclicos: la Doxepina, por ejemplo, es un sedante muy afín a los receptores de histamina H1, efectivo para mantener el sueño y disminuir los despertares nocturnos (15).

\section{COMPLICACIONES}

El insomnio crónico tiene un impacto adverso en la actividad diurna y en la calidad de vida. Al estar relacionado con la actividad del sistema nervioso simpático, se ha demostrado su asociación con un riesgo cardiovascular elevado, hipertensión, infarto al miocardio, diabetes y síndrome metabólico $(16,17)$.

Los síntomas de insomnio se han asociado con un mayor riesgo de ideación y comportamientos suicidas. Esta asociación muchas veces está mediada por una depresión subyacente, pero otros posibles factores contribuyentes incluyen: disfunción serotoninérgica, disfunción cognitiva, medicamentos y abuso de sustancias $(7,18)$. 
Los pacientes con insomnio en general reportan aumento de fatiga, somnolencia, confusión, tensión, ansiedad y en general una disminución significativa en su felicidad $(2,4,9)$.

\section{CONCLUSIONES}

El insomnio es una patología con una alta prevalencia en la población general, predominantemente en mujeres y adultos mayores, esta prevalencia aumenta en pacientes con trastornos psiquiátricos subyacentes, por lo que la historia clínica y la búsqueda de factores de riesgo y comorbilidades se vuelve clave para el adecuado diagnóstico. Su clasificación se basa en el tiempo de evolución, gravedad y en la identificación de causas subyacentes.

El tratamiento exitoso requiere la atención tanto al insomnio en sí, como de las comorbilidades relevantes por lo que se deben tener presentes dentro del diagnóstico diferencial otros trastornos del sueño comunes como la apnea obstructiva, las alteraciones en el ciclo circadiano y los trastornos del sueño relacionados con el movimiento.

La literatura sugiere como primera elección el manejo no farmacológico, basado en higiene del sueño, control de estímulos y técnicas de relajación. En algunos casos seleccionados se elige tratamiento farmacológico como coadyuvante a la terapia no farmacológica, el cual debe ser individualizado para cada paciente, siendo las benzodiacepinas, los compuestos $Z$, los agonistas del receptor de melatonina y los antidepresivos tricíclicos los principales medicamentos utilizados.

El insomnio tiene un impacto significativo en la calidad de vida de los pacientes y se ha asociado a un incremento en el riesgo cardiovascular, diabetes, síndrome metabólico, suicidio y mayor mortalidad.

Se recomienda a los médicos estudiar el tema debido a la alta frecuencia con que se presentan pacientes con este padecimiento, es importante dar un seguimiento al paciente para establecer el tratamiento que mejor se adapte y funcione. Según la bibliografía consultada, no se conoce exactamente el aumento en prevalencia de este padecimiento en tiempos de pandemia, pero se deduce por la investigación realizada, que el porcentaje de pacientes con insomnio aumentará, debido a que el estrés es un factor de riesgo previamente identificado.

\section{REFERENCIAS BIBLIOGRÁFICAS}

1. Grau-López L,et al. Prevalencia administrativa del insomnio en pacientes adictos durante el consumo activo de las sustancias y características clínicas asociadas.. Acta Española Psiquiátrica. 2016; 44((2) 64-71). ISSN 1139-9287

2. Sheng Zhi Zhao, et al. Short sleep duration and insomnia symtoms were associated with lower happiness levels in Chinese adults in Hong Kong. Int. J. Environ. Res. Public Health. 2019;(16 2079 - 2090.). doi: https://doi.org/10.3390/ijerph16122079

3. Blanco W, et al. Synaptic Homeostasis and Restructuring across the Sleep-Wake Cycle. PLoS Comput Bio. 2015; 27/3: 247-257). https://doi.org/10.1371/journal.pcbi.1004241

4. Katarzyna Szmyd J,WA. The influence of circadian rhythm of sleep on functioning in a marital relationship and on a sexual activity. Adv Psychiatry Neurol. 2018; 27((3): 247-257.). doi: https://doi.org/10.5114/ppn.2018.78717

5. Guidozzi F. Gender differences in sleep in older men and women.. Climacteric.. 2015; 18(715-721). doi: https://doi. org/10.3109/13697137.2015.1042451

6. Selsick H,OD. Sleep disordes in phychiatry.. BJPsych Advances.. 2018;(24, 273 -283.). doi: https://doi.org/10.1192/ bja.2018.8

7. Bonnet M,AD. Evaluation and diagnosis of insomnia in adults.. UpToDate. 2019. 2019;(Topic 7676 Version 32.0). https://www-uptodate-com.ezproxy.sibdi.ucr.ac.cr/contents/risk-factors-comorbidities-and-consequences-of-insomnia-in-adults/print?search=insomnio\&topicRef $=76 \%$ E2\%80\%A6

8. Chávez $M$, et al. En búsqueda del hipnótico ideal: tratamiento farmacológico del insomnio. AVFT. 2017; 36(( 1 ): 10-21.). https://www.researchgate.net/publication/314236912_En_busqueda_del_hipnotico_ideal_tratamiento_farmacologico_del_insomnio

9. Raman S,RA. Insomnia - A general review.. Drug invention Today.. 2019; 12((1) 123-126.). ISSN: 0975-7619 
10. Madronal M,FF. Protocolo terapéutico de las alteraciones del sueño en el anciano. Protocolos de práctica asistencial. Medicine. 2015; 11((84): 5049 - 53). DOI: https://doi.org/10.1016/j.med.2015.07.016

11. Brasure M, et al. Management of Insomnia Disorder. Agency for Healthcare Research and Quality. 2015;(159). https://www.ncbi.nlm.nih.gov/books/NBK343503/

12. Buysee D, et al. Clinical Management of Insomnia. JAMA. 2017; 318(20). doi: https://doi.org/10.1001/ jama.2017.15683

13. Salisbury-Afshar E. Management of Insomnia Disorder in Adults. AHRQ. 2018; 98((5); 319- 323). https://www.aafp. org/afp/2018/0901/p319.html

14. Qaseem A, et al. Management of Chronic Insomnia Disorder in Adults: A Clinical Practice Guideline From the American College of Physicians.. Ann Intern Med. 2016; 165((2) 125-133). doi: https://doi.org/10.7326/M15-2175

15. Lie JD, et al. Pharmacological Treatment of Insomnia. P \& T. 2015; 40((11), 759-771). PMID: 26609210

16. Bertisch $S M$, et al. Insomnia with objective short sleep duration and risk of incident cardiovascular disease and all-cause mortality: Sleep Heart Health Study. Sleep 2018; 41. doi: https://doi.org/10.1093/sleep/zsy047

17. Fernandez-Mendoza J, et al. Impact of the Metabolic Syndrome on Mortality is Modified by Objective Short Sleep Duration. J Am Heart Assoc 2017; 6. doi: https://doi.org/10.1161/JAHA.117.005479

18. Woznica $A A$, et al. The insomnia and suicide link: toward an enhanced understanding of this relationship. Sleep Med Rev 2015; 22:37. doi: https://doi.org/10.1016/j.smrv.2014.10.004

19. Igoche D, HA, et al. Sleep Pattern and Sleep Hygiene Practices among Nigerian Schooling Adolescents.. Indian J Psychol Med.. 2017;(39:407-12.). doi: https://doi.org/10.4103/0253-7176.211743 